\title{
Orbital sporadic Burkitt lymphoma in an adult diabetic African American female and a review of adult orbital cases
}

This article was published in the following Dove Press journal:

Clinical Ophthalmology

19 April 201 I

Number of times this article has been viewed

\author{
John Carmody' \\ Raghunath P Misra ${ }^{1,2}$ \\ Marlyn P Langford' \\ William A Byrd' \\ Lauren Ditta' \\ Bryan Vekovius' \\ Donald E Texada'
}

'Department of Ophthalmology, ${ }^{2}$ Department of Pathology, Louisiana

State University Health Sciences

Center, Shreveport, LA, USA
Correspondence: Marlyn P Langford Department of Ophthalmology, Louisiana State University Health Sciences Center, I50I Kings Hwy, PO Box 33932,

Shreveport, LA 7II30-3932, USA

$\mathrm{Tel}+\mathrm{I} 3186755018$

Fax +13186756000

Email mlangf@lsuhsc.edu

\begin{abstract}
A case of sporadic Burkitt lymphoma (sBL) presenting with jaw and lid involvement in a diabetic adult African American female and a review of adult orbital Burkitt lymphoma cases are presented. Lid edema, visual loss, ophthalmoparesis, proptosis, and sinusitis progressed over 4 weeks despite antibiotic and steroid treatment. Upper lid biopsy histopathological evaluation and immunophenotyping revealed a homogenous mass of atypical CD10 and CD20-negative B-cells and tingible body macrophages yielding a "starry sky" appearance. Cytogenetic analysis detected a minor variant c-MYC translocation, but no Epstein-Barr virus RNA. Detection of multiple lesions prompted a diagnosis of stage IV disease that totally regressed following radiation and chemotherapy. Review results of the six adult orbital sBL cases support a poor prognosis and a heightened suspicion of variant CD10, CD20 and BCL6 positive sBL in adults presenting with jaw pain and rapidly progressive orbital symptoms, particularly in female, African American, and diabetic patients.
\end{abstract}

Keywords: B-cells, Burkitt lymphoma, cancer, diabetes, eye, Epstein-Barr virus, orbit, tumor

\section{Introduction}

Over 50 years ago Denis Burkitt described a monoclonal lymphoreticular cell tumor that bears his name. ${ }^{1-3}$ His contributions led to the discovery of the Epstein-Barr virus (EBV; human lymphotropic herpes virus), ${ }^{4}$ EBV's relationship to Burkitt lymphoma (BL) and other lymphomas, and other oncogenic viruses. ${ }^{5,6} \mathrm{BL}$, originally described as a jaw mass seen in male children residing in equatorial Africa, ${ }^{1,2}$ commonly presents with extralymphnodal lesions; usually in the abdomen, mediastinum, spinal cord, gonads, orbit, salivary glands, urogenital tract, and/or bone marrow. BL is composed of malignant noncleaved B-lymphocytes and is highly aggressive with a doubling time of about 24 hours. ${ }^{78} \mathrm{BL}$ occurs in all age and racial groups throughout the world. ${ }^{9-13}$

Three subtypes of BL are described based upon their epidemiological distribution: endemic (eBL); sporadic (sBL); and immunodeficiency-associated (i-aBL). All BL subtypes share morphological, growth, and immunophenotypic characteristics and a nonrandom genetic heterogeneity. ${ }^{14,15}$ That is, BL arises due to reciprocal chromosomal translocations of the c-MYC proto-oncogene with different immunoglobulin heavy chain (IGH) genes resulting in c-MYC gene overexpression leading to monoclonal expansion of immature B cells. ${ }^{15,16}$ Several other chromosomal abnormalities are present in BL from EBV-positive children, but their roles are unknown. ${ }^{14,17,18}$ Endemic BL usually presents in EBV-seropositive children as an extranodal tumor usually involving the jaw and soft tissues of the orbit and abdomen in male children and young adults that reside in high-incidence (malnutrition, EBV and malaria infestation) 
zones of equatorial Africa and South America (50-100 cases/million/year). ${ }^{1,2,9,11,12,19}$ Pediatric and adult sBL cases occur worldwide in countries outside the equatorial zone and commonly present with tumor involvement. ${ }^{9,12,13,19}$ The incidence of sBL in the United States is 2-3 cases/million/ year. ${ }^{9}$ Sporadic BL accounts for $30 \%-50 \%$ of lymphomas in children, but only $1 \%-2 \%$ of lymphomas in adults. ${ }^{11,20,21}$ In contrast to eBL, sBL rarely presents as orbital disease in United States children. ${ }^{22,23}$ Immunodeficiency-associated BL occurs in immunocompromised patients, such as AIDS patients and organ transplant recipients. Immunodeficiencyassociated BL accounts for $20 \%-35 \%$ of lymphomas in pediatric and adult AIDS cases and the clinical features are similar to those of sBL, except for a much higher initial involvement of the central nervous system (CNS). ${ }^{16,21,24-28}$ c-MYC rearrangements and EBV infection are detectable in $20 \%-40 \%$ of HIV-associated cases. ${ }^{29}$ In AIDS-associated $\mathrm{i}-\mathrm{aBL}$, defective regulation of EBV-infection is thought to give rise to uncontrolled B-cell differentiation. ${ }^{30} \mathrm{~A} \mathrm{BL}$ variant of post-transplant lymphoproliferative disorder (PTLD) can occur in solid organ transplant recipients who receive long-term immunosuppressive therapy and is usually characterized by weight loss, fever, and generalized lymphadenopathy with bone marrow involvement, ${ }^{31-33}$ c-MYC rearrangement and type A EBV infection in $>80 \%$ of cases. ${ }^{32-34}$ In PTLD patients, the interaction of the latent EBV nuclear antigen with the BCL2 gene prevents apoptosis and promotes B-cell immortalization. ${ }^{17}$

Lymphomas of the ocular adnexa represent $8 \%-11 \%$ of all extranodal lymphoid tumors. ${ }^{20,35-37}$ The sporadic form of BL occurs primarily in "older" American adults (most $>40$ years old). ${ }^{9}$ Orbital involvement as the presenting symptom in adult sBL is rare; PubMed and OVID electronic searches identified five adult orbital sBL cases. ${ }^{38-41}$ Herein we report a case of orbital sBL in an African American female adult who presented with jaw pain, lid edema, and new onset diabetes mellitus (DM) and briefly review the adult orbital sBL cases. Orbital sBL symptoms may masquerade initially as an infection (cellulitis) or inflammatory pseudotumor delaying diagnosis, staging and appropriate therapy. Our results support a heightened suspicion of sBL in adults presenting with jaw pain and rapidly progressive orbital symptoms, particularly in female, African American, and diabetic patients.

\section{Case report}

A 49-year-old African American female presented to a local dentist in late December with right-side tooth pain.
The dentist empirically prescribed Cefadroxil and Loritab based upon a negative X-ray of the right jaw and advised her to seek emergency care if symptoms worsened. Four days later, she presented at a local Emergency Room and was admitted to our hospital with complaints of rightside "teeth pain", numbness of upper and lower lips, and edema of upper eyelid. Her past medical history was significant for penicillin and sulfa allergies, foot surgery and a hysterectomy. The patient's consultant ophthalmic evaluation revealed a best corrected visual acuity of 20/30 OD and $2+$ to $3+$ upper eyelid edema that was not warm or tender to touch. She had normal extraocular movement, pupillary reactions, intraocular pressures and ocular fundi of the affected and fellow eyes. No other ocular symptoms were noted. Computed tomography (CT) scans of the orbit revealed extensive soft tissue swelling in the right paraseptal orbit and mucosal thickening in the right frontal and maxillary sinuses, and right anterior and medial ethmoidal cells (Figure 1A) consistent with a presumptive diagnosis of preseptal cellulitis. The patient received a 5-day course of intravenous antibiotics (Vancomycin and Clindamycin) with improvement in jaw pain, but only slight improvement
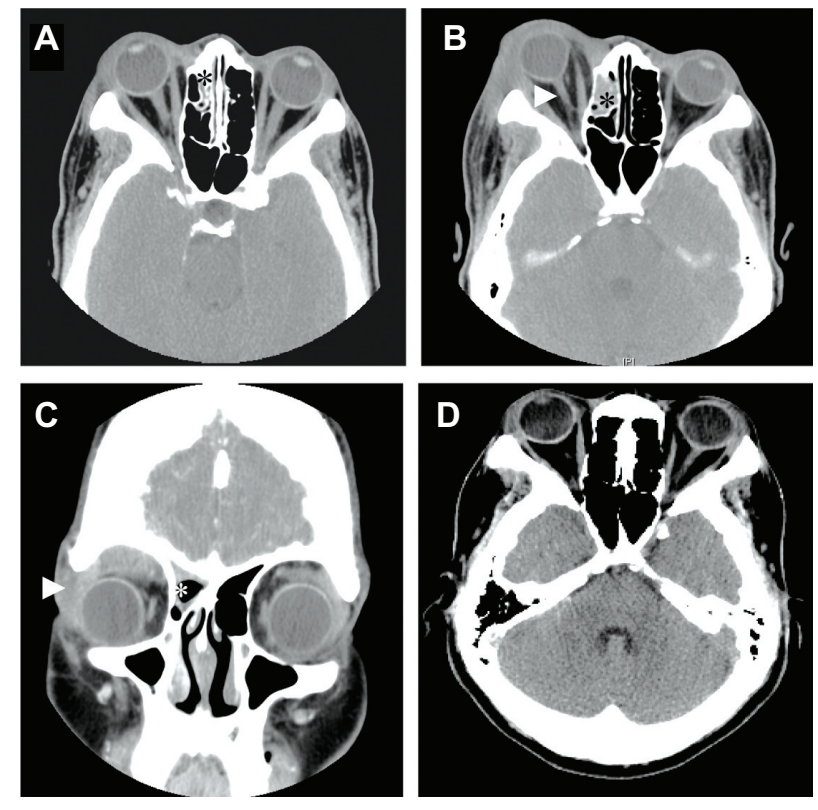

Figure I A) Axial computed tomography (CT) scan showing extensive soft tissue swelling and mucosal thickening of right ethmoidal sinus (*). B) Axial CT scan (6 days after the first CT scan) showing increased enlargement of the extraconal soft tissue mass in the right orbital roof with inferior displacement of the globe. Note the marked proptosis and "banjo-string" deformation of the optic nerve (arrowhead) and increased mucosal thickening in the right maxillary and frontal sinuses (asterisks in scans). C) Coronal reconstruction view (6 days after the first CT scan) supported extension of the soft tissue mass in the superior lateral right orbit (arrowhead) as well as ethmoid sinusitis (*). D) Axial CT scan shows normal ocular presentation with no evidence of tumor in the orbit or sinus at 21 months post-initiation of treatment. 
in orbital symptoms. Her clinical work-up was significant for hypertension and new onset diabetes. Accordingly, the patient's comprehensive metabolic panel was significant for elevated glucose in plasma (273 to $321 \mathrm{mg} / \mathrm{dL}$ ) and urine $(1000 \mathrm{mg} / \mathrm{dL})$, as well as slightly elevated ketones $(5 \mathrm{mg} / \mathrm{dL})$ in her urine. Her CBC was significant for elevated erythrocytes $(5.25$ million $/ \mu \mathrm{L})$ and hemaglobin $(15.6 \mathrm{~g} / \mathrm{dL})$. She was discharged on hypertension and DM control medications, diabetic diet, prednisone ( $40 \mathrm{mg}$ /day for 1 day, then $20 \mathrm{mg} /$ day for 3 days) for treatment of the suspected noninfectious pseudotumor. She was given a follow-up eye clinic appointment (1 week) and instructed to return sooner if symptoms worsened.

The patient presented to the LSUHSC Eye Clinic six days later with complaints of progressive lid edema and proptosis. An external eye examination revealed decreased vision (20/60 OD), restricted extraocular motion ( -2 in up gaze/down gaze, -1 in right/left gaze), 3+ edema of right upper eyelid (not warm or tender-to-touch), and conjunctival chemosis. CT scans revealed increased swelling of soft tissue of the right lid, mucosal thickening of right frontal and maxillary sinuses, and proptosis (Figure 1B). Comparison of CT scans confirmed an aggressively expanding extraconal mass undifferentiated from the lacrimal glands, causing a stretched optic nerve yielding a "banjo string" sign, and downward displacement of the globe (Figure 1C).

The patient consented to a right upper lid biopsy, and it was performed on day 21 after initial presentation. The histopathological evaluation revealed a homogenous mass infiltrating the soft orbital tissue (Figure 2A) composed of medium sized noncleaved cells with nuclei containing multiple nucleoli and a thin rim of basophilic cytoplasm. Also, the mass contained scattered histiocytic cells (macrophages

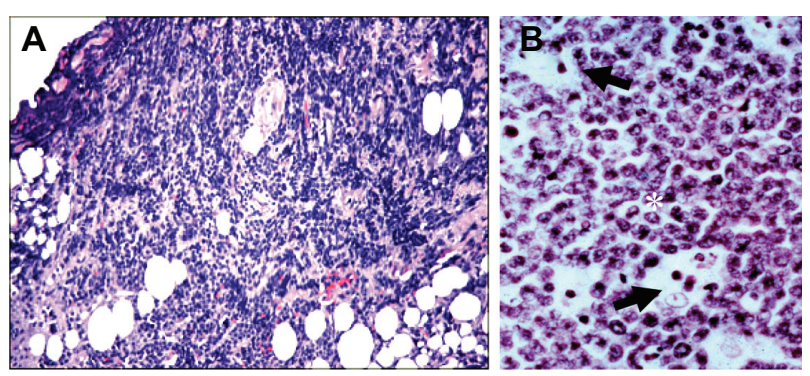

Figure 2 A) Histopathology of the lid biopsy tissue showing inflammation and invasion of the lid tissue by large lymphocytes. B) High power magnification of the biopsy revealing the typical appearance of BL characterized by the tightly packed proliferative neoplastic lymphocytes $(*)$ with high nuclear-to-cytoplasmic ratios interspersed with histiocytic cells (large tingible body macrophages) containing dead cell debris (arrows) yielding a "starry sky" appearance (ie, stars in a sky of B cells). (H\&E; 400×). containing phagocytized cellular debris) yielding a "starry sky" pattern consistent with a lymphoproliferative disorder, possibly high-grade lymphoma (Figure 2B). Immunophenotyping and cytogenetic analyses supported a diagnosis of BL. Immunohistochemistry of the biopsied tissue showed positive staining for CD19, CD22, CD24, CD30, CD79A (immunoglobulin-associated $\alpha$ antigen), and cytoplasmic $\kappa$-light chain, but negative staining for CD3, CD10, CD20, BCL11, BCL14, and TdT. Cytogenetic evaluation using fluorescence in situ hybridization (FISH) probes designed to detect the most frequently observed translocation $\mathrm{t}(8 ; 14)$ (involving MYC region at 8Q24 and IGH at 14Q32) was negative, but using a mixture of two probes that detect other variant MYC translocations revealed a c-MYC rearrangement consistent with a minor variant MYC translocation at either $\mathrm{t}(8 ; 22)$ or $t(2 ; 8) .{ }^{14-16}$ The patient's BL did not contain detectable EBV RNA. Flow cytometry demonstrated monoclonal B-cell expansion consistent with BL. The patient's serum lactate dehydrogenase (LDH) was elevated at $826 \mathrm{IU} / \mathrm{dL}$. The patient's systemic work-up included a proton emission tomography (PET) scan, performed on day 24 post onset of symptoms, that revealed a systemic disease process with bone involvement of the mid and upper thoracic spines and brain. Lumbar puncture and bone marrow aspirates were negative for BL cells. Concomitantly, CT scans of the chest, abdomen and pelvis revealed subcentimeter axillary nodes, small soft tissue lesions involving thoracic vertebrae and thoracic descending aorta, small bilateral focal renal lesions and heterogeneous abnormalities in the presacral region and right lateral wall of urinary bladder.

Our patient received a 4-week course of daily radiation therapy and underwent placement of an Omaya reservoir in the ventricular system of the brain 1 week into the radiation therapy to facilitate intrathecal chemotherapy. Her chemotherapy regime included four cycles of high dose cyclophosphamide, intrathecal methotrexate and cytarabine. After 10 months of radiation and chemotherapy treatment, CT scans showed complete resolution of the ocular disease (Figure 1D). The patient is in remission with full ocular function at 5 years after treatment. The LSUHSC IRB/Ethics Committee ruled that approval was not required for this study.

\section{Discussion}

The presenting symptoms of jaw pain and orbital involvement in our 49-year-old diabetic African American female patient are commonly observed in African children with eBL. That 
is, about $65 \%$ of African children with eBL present with concurrent jaw and orbital symptoms. ${ }^{11}$ Our case is the second adult sBL patient to present with jaw and facial symptoms. ${ }^{41}$ Only six confirmed adult orbital sBL cases (average age of $61 \pm 22$ years; range 24 to 84 years) are reported in the literature to date (Table 1). Half or more of the adult orbital sBL patients are aged $\geq 40$ years and presented with or developed facial swelling, orbital edema, loss of vision, proptosis, restricted ocular motility, chemosis, and sinus involvement. Notably, 66\% (2 of 3) of the African American adult orbital sBL cases presented with facial pain, orbital edema, proptosis, and sinus involvement. Comparison of the reported adult $\mathrm{sBL}$ cases indicates females are affected more often than males $(2: 1)$, but did not reveal a race or eye predilection. The 3 African American, 2 Caucasian and 1 Chinese adult orbital sBL presented with an equal distribution between left and right eyes (3:3).

Misdiagnosis of orbital sBL is common due to the rare presentation of ocular involvement. As in our case and most of the previously reported adult sBL cases, ${ }^{38-41}$ making the correct diagnosis can be difficult; the process being one of exclusion. Accordingly, most of the adult sBL patients received antibiotics and/or steroids initially for suspected infectious and inflammatory diseases with transient or no improvement. The progressive growth over a 2-week period was highly suggestive of BL and consistent with the rapid growth observed in most lymphomas..$^{7-9,19,36-38}$ Once BL was suspected, PET and CT scans revealed multiple systemic BL lesions consistent with a stage IV disease. Most of the adult orbital sBL patients (4 of 6) had multiple extranodal lesions in bone marrow, brain, heart, chest, abdomen, pelvis, and/or groin, but two patients had orbital lesions only (Table 1). Since $88 \%$ (14 of 16) of the adult orbital BL patients had systemic involvement, ${ }^{41}$ it is recommended that all suspected adult orbital BL patients be given emergent systemic evaluations. Regression of our patient's BL with complete recovery of vision occurred following combination treatment with radiation, intrathecal and standard systemic chemotherapy. ${ }^{41}$ Review of the adult orbital sBL case outcomes revealed death and/or persistent lesions in $83 \%$ (5 of 6 ) of patients at 1 year; three of the six patients died within 10 months of the initial diagnosis, two patients had total regression of the orbital lesion but systemic BL persisted, while our patient remains free of BL after 5 years (Table 1). These results are consistent with the high mortality reported in older BL patients, ${ }^{41}$ but firm numbers are not available since few therapeutic trials include BL patients aged over 60 years. Several factors may be responsible for the high mortality. The treatment response appears to vary with age, extent of disease (especially CNS involvement), immunophenotype and translocation genotype. ${ }^{33,35,38,41}$ Further, i-aBL patients generally require modifications of radiation and chemotherapy. ${ }^{16,19,33}$ Safe and effective antiviral and immuno-therapies to prevent EBV latency and EBV-associated lymphomas are under investigation. $^{42}$

A diagnosis of BL is confirmed by tumor histopathological examination and immunophenotyping. The histopathological examinations of our patient's lid biopsy revealed a mass of noncleaved lymphocytic cells with scattered tingible body macrophages containing phagocytized cellular debris yielding a "starry sky" appearance. Evaluations of all tissue biopsies from the adult orbital BL cases yielded histopathological findings consistent with a diagnosis of BL (Table 1). The histopathology descriptions of four of the six adult sBL tumor biopsies noted a "starry sky" appearance. The classical BL immunophenotypic profile includes B-cell markers CD10, CD20, CD21 (common to endemic BL), CD79a, BCL-6 (downregulation of BCL6 required for plasmacyte differentiation $)^{43}$ and IgM, but not CD5, CD23, TdT and BCL-20. ${ }^{7,11,32,36}$ A comparison of immunophenotype profiles of five adult orbital sBL cases revealed $80 \%$ (4 of 5 cases) expressed B-cell markers CD10 and CD20, 60\% (3 of 5) expressed BCL6 (B-cell CLL/lymphoma), 40\% (2 of 5) expressed CD79A (immunoglobulin-associated alpha), and $40 \%$ ( 2 of 5 ) expressed Ki67. Immunophenotypic analysis of our patient's biopsied tissue showed positive staining for BL cell markers CD19, CD22, CD24, CD30, and CD79a. Our patient's BL did not react with CD10 (common acute leukemia/lymphoma antigen) or CD20 (membrane spanning domain antigen). Decreased CD20 expression is common to some high-grade aggressive B-cell lymphomas with translocations involving c-MYC and BCL6. ${ }^{44}$

Review of the adult orbital sBL cases revealed 50\% (3 of 6) of the patients had DM and 67\% (2 of 3) of the diabetic orbital sBL expressed BCL6 (Table 1). The high incidence of orbital sBL associated with DM suggests a theoretical association. Although an increased incidence of several cancers has been observed in diabetic patients ${ }^{45,46}$ and a role for ketone and lactate in tumor growth and metastasis has been postulated recently, ${ }^{47}$ an epidemiologic basis or molecular mechanism for an association between human EBV infection, orbital sBL and diabetes lacks direct support at present. It is noteworthy that increased diabetes susceptibility and diabetic autoimmune disease in experimental animal models are associated with altered BCL genes. ${ }^{48,49}$ Concomitantly, the EBV load is slightly higher in type $1 \mathrm{DM}$ patients than in 


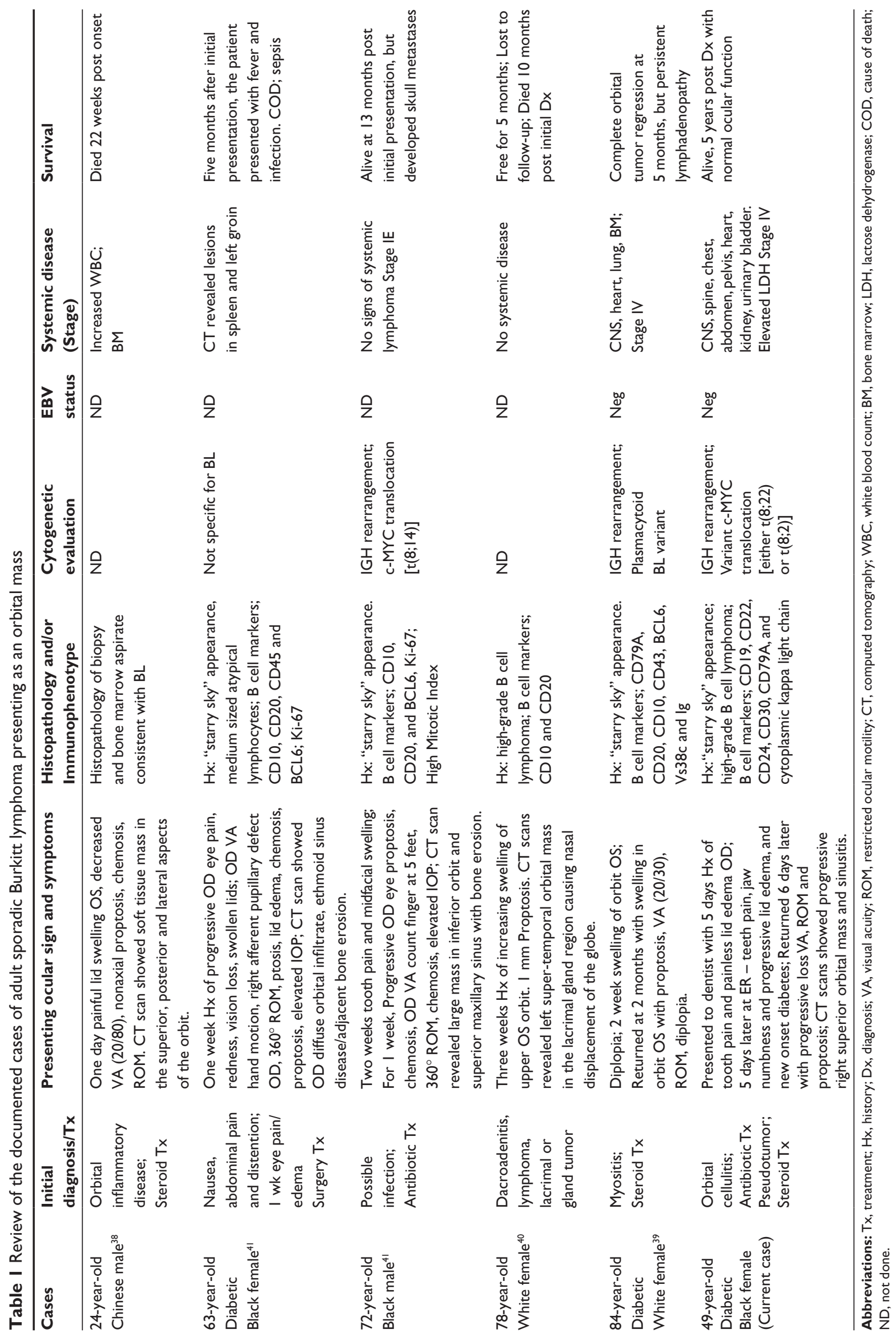


non-DM patients. ${ }^{50}$ Further, EBV nuclear antigen can interact with BCL genes. ${ }^{51}$ A BCL6 translocation is suspected in our patient due to the reported association between c-MYC and BCL translocations and decreased CD20. ${ }^{44}$ However, BCL6 gene translocation or expression analyses were not obtained on our patient's BL. Additional studies are needed to investigate EBV-associated cMYC and BCL translocations in adult diabetic patients with and without sBL. It should be noted that EBV infection and c-MYC translocations involving IGH and BCL genes are not exclusive to BL, being found in other B-cell lymphomas. ${ }^{19-21,36,44}$

Most BL (80\%) have a translocation of the c-MYC proto-oncogene from chromosome 8 to the IGH gene region of chromosome $14 \mathrm{~b}[\mathrm{t}(8 ; 14)]$ and most $\mathrm{BL}$ patients have a history of EBV infection. In fewer cases $(15 \%-20 \%)$, the c-MYC gene is translocated to the $\kappa$-light chain loci on chromosome $2[\mathrm{t}(8 ; 2)]$ or to the $\lambda$-light chain on chromosome $22[\mathrm{t}(8 ; 22)] \cdot{ }^{14-16,18,19} \mathrm{FISH}$ analysis of our patient's orbital mass did not detect either of the classical translocations. FISH analysis using an alternate probe for segregation of c-MYC showed fission of the c-MYC locus and supported a minor variant translocation involving chromosome 2 or 22 . While the detection of a c-MYC translocation strongly supports the diagnosis of $\mathrm{BL}$, demonstration of a c-MYC rearrangement in the context of other clinico-pathologic findings in our patient is confirmatory. Cytogenetic evaluation identified c-MYC gene rearrangements involving IGH genes in 3 of 4 adult orbital BL analyses, but the translocations appear to have involved different gene loci (Table 1). Interestingly, only one of the orbital sBL had a classical c-MYC translocation $[\mathrm{t}(8 ; 14)] \cdot{ }^{41}$ Moreover, comparisons of the cytogenetic results failed to identify a consensus IGH gene rearrangement involving the c-MYC gene in 4 adult orbital sBL. The failure to detect EBV RNA in our patient's BL cells is consistent with the generally low frequency of EBV detection $(25 \%-30 \%)$ in European and North/South American sBL cases. ${ }^{9}$ Similarly, EBV RNA was not detected in two adult orbital sBL cases (Table 1). In contrast, EBV is detected in 98\% eBL, 30\%-40\% HIV-associated BL, and $80 \%-100 \%$ of the BL variant of PTLD. ${ }^{11,17,18,29,32}$

In summary, we present a rare case of adult orbital $\mathrm{sBL}$ that masqueraded initially as cellulitis/pseudotumor, but responded to radiation and chemotherapy once correctly diagnosed. The results of the review of the six adult orbital sBL cases was remarkable for 2:1 female-to-male predilection; a history of diabetes; B-cells expressing CD10, CD20 and BCL6; and an initial clinical presentation of jaw and orbital symptoms in adult African Americans.

\section{Acknowledgement}

The authors thank Christopher Duggan for excellent technical assistance.

\section{Disclosure}

The authors report no conflicts of interest in this work.

\section{References}

1. Burkitt D. A sarcoma involving the jaws in African children. Br J Surg. 1958;46:218-223.

2. Burkitt D, O'Connor GT. Malignant lymphoma in African children. I. A clinical syndrome. Cancer. 1961;14:258-269.

3. Coakley D. Denis Burkitt and his contribution to haematology/oncology. Br J Haematol. 2006;135:17-25.

4. Epstein M, Achong B, Barr Y. Virus particles in cultured lymphoblasts from Burkitt's lymphoma. Cancer. 1964;1:702-703.

5. De-Thé G, Geser A, Day NE, et al. Epidemiological evidence for a causal relationship between Epstein-Barr virus and Burkitt's lymphoma from Ugandan prospective study. Nature. 1978;274:756-761.

6. Wong M, Pagano JS, Schiller JT, et al. New associations of human papillomavirus, simian virus 40, and Epstein-Barr virus with human cancer. J Natl Cancer Inst. 2002;94:1832-1836.

7. Ziegler JL. Burkitt's lymphoma. N Eng J Med. 1981;305:735-745.

8. Magrath I. The pathogenesis of Burkitt's lymphoma. Adv Cancer Res. 1990;55:143-144.

9. Levine PH, Kamaraju LS, Connelly RR, et al. The American Burkitt's lymphoma registry: eight years' experience. Cancer. 1982;49: 1016-1022.

10. Garrity JA, Henderson JW. Orbital Tumors. 4th Edition, Philadelphia, PA: Lippincott, Williams \& Wilkins; 2007:251-253.

11. Odeoye AO, Durosinmi MA, Adeodu OO, et al. Ocular manifestations of Burkitt's lymphoma: experience in Ile-Ife South Western Nigeria. West Afr J Med. 2007;26:48-52.

12. Parkin M, Nectoux J, Stiller C, Draper G. L'incidence des cancers de l'enfant dans le monde. Pediatrie. 1989;44:725-736.

13. Banthia V, Jen A, Kacker A. Sporadic Burkitt's lymphoma of the head and neck in the pediatric population. Int J Pediatr Otorhinolaryngol. 2003;67:59-65.

14. Neri A, Barriga F, Knowles DM, et al. Different regions of the immunoglobulin heavy-chain locus are involved in chromosomal translocations in distinct pathogenic forms of Burkitt lymphoma. Proc Natl Acad Sci USA. 1988;85:2748-2752.

15. Toujani S, Dessen P, Ithzar N, et al. High resolution genome-wide analysis of chromosomal alterations in Burkitt's lymphoma. PLoS One. 2009;4:1-14.

16. Jaffe ES. The 2008 WHO classification of lymphomas: implications for clinical practice and transitional research. Hematology Am Soc Hematol Educ Program. 2009:523-531.

17. Pasquale MA, Weppler D, Smith J, et al. Burkitt's lymphoma variant of post-transplant lymphoproliferative disease (PTLD). Pathol Oncol Res. 2002;8:105-108.

18. Gutierrez MI, Bhatia K, Barriga F, et al. Molecular epidemiology of Burkitt's lymphoma from South America: differences in breakpoint location and Epstein-Barr virus association from tumors in other world regions. Blood. 1992;79:3261-3266.

19. Perkins AS, Friedberg JW. Burkitt lymphoma in adults. Hematology Am Soc Hematol Educ Program. 2008;341-348.

20. Wong MB, Strasnick B, Zimmerman MC. Extranodal American Burkitt's lymphoma of the head and neck. Arch Otolaryngol Head Neck Surg. 1992;118:193-199.

21. Harris NL, Horning SJ. Burkitt's lymphoma - the message from microarrays. N Engl J Med. 2006;354:2495-2498.

22. Weisenthal RW, Streeten BW, Dubansky AS, et al. Burkitt lymphoma presenting as a conjunctival mass. Ophthalmology. 1995;102: 129-134. 
23. Edelstein C, Shields JA, Shields CL, et al. Non-African Burkitt lymphoma presenting with oral thrush and an orbital mass in a child. Am J Ophthalmol. 1997;124:859-861.

24. Iordānescu C, Mătuşa R, Denislam D, et al. The ocular manifestations of AIDS in children. Oftalmologia. 1993;37:308-314.

25. Nadal D, Caduff R, Frey E, et al. Non-Hodgkin's lymphoma in four children infected with the human immunodeficiency virus. Association with Epstein-Barr Virus and treatment. Cancer. 1994;73:224-230.

26. Robinson MR, Sailt RB, Bryant-Greenwood PK, et al. Burkitt's/ Burkitt's-like lymphoma presenting as bacterial sinusitis in two HIV-infected children. AIDS Patient Care STDS. 2001;15: 453-458.

27. Brooks HL Jr, Downing J, McClure JA, Engel HM. Orbital Burkitt's lymphoma in a homosexual man with acquired immune deficiency. Arch Ophthalmol. 1984;102:1533-1537.

28. Levy J, Kratz A, Lifshitz T. Burkitt's lymphoma presenting as oculomotor palsy in an HIVpositive patient. Eur J Ophthalmol. 2006; 16:186-189.

29. Brady G, MacArthur GJ, Farrell PJ. Epstein-Barr virus and Burkitt lymphoma. J Clin Pathol. 2007;60:1397-1402.

30. Birx DL, Redfield RR, Tosato G. Defective regulation of Epstein-Barr virus infection in patients with AIDS or AIDS-related disorders. $N$ Eng J Med. 1986;314:874-879.

31. Liu GT, Kay MD, Byrne GE, et al. Ophthalmoparesis due to Burkitt's lymphoma following cardiac transplantation. Neurology. 1993;43: 2147-2149.

32. Gong JZ, Stenzel TT, Bennett ER, et al. Burkitt lymphoma arising in organ transplant recipients: a clinicopathologic study of five cases. Am J Surg Pathol. 2003;27:818-827.

33. Schubert S, Abdul-Khaliq H, Lehmkuhl HB, et al. Diagnosis and treatment of post-transplantation lymphoproliferative disorder in pediatric heart transplant patients. Pediatr Transplant. 2009;13:54-62.

34. Frank D, Cesarman E, Lie YF, et al. Post-transplantation lymphoproliferative disorders frequently contain Type A and not Type B Epstein-Barr virus. Blood. 1995;85:1391-1403.

35. Freeman C, Berg JW, Cutler SJ. Occurrence and prognosis of extranodal lymphomas. Cancer. 1972;29:252-260.

36. Coupland SE, Krause L, Delecluse H-J, et al. Lymphoproliferative lesions of the ocular adnexa. Analysis of 112 cases. Ophthalmology. 1998;105:1430-1441.

37. Shields JA, Shields CL, Scartozzi R. Survey of 1264 patients with orbital tumors and simulating lesions. The 2002 Montgomery Lecture, Part 1. Ophthalmology. 2004;111:997-1008.
38. Au Eong KG, Choo CT. Burkitt lymphoma manifesting as acute proptosis. Am J Ophthalmol. 1997;123:856-858.

39. Coupland SE, Hummel M, Stein H. Ocular adnexal lymphomas: five case presentations and a review of the literature. Surv Ophthalmol. 2002;47:470-490.

40. Sandy CJ, Rose GE, Clark BJ, Plowman PN. Sporadic Burkitt's lymphoma presenting as solely orbital disease in a 78-year-old. Eye. 2001;15:113-115.

41. Baker PS, Gold KG, Lane KA, et al. Orbital Burkitt lymphoma in immunocompetent patients: a report of 3 cases and a review of the literature. Ophthal Plast Reconstr Surg. 2009;25:464-468.

42. Cader FZ, Kearns P, Young L, et al. The contribution of the EpsteinBarr virus to the pathogenesis of childhood lymphomas. Cancer Treat Rev. 2010;36:348-353.

43. Diehl SA, Schmidlin H, Nagasawa M, et al. STAT3-mediated upregulation of BLIMP1 is coordinated with BCL6 down-regulation to control human plasma cell differentiation. J Immunol. 2008;180: 4805-4815.

44. Wu D, Wood BL, Dorer R, Fromm JR. "Double-hit" mature B-cell lymphomas show a common immunophenotype by flow cytometry that includes decreased CD20 expression. Am J Clin Pathol. 2010; 134:258-265.

45. Grote VA, Becker S, Kaaks R. Diabetes mellitus type-2: an independent risk factor for cancer? Exp Clin Endocrinol Diabetes. 2010;118:4-8.

46. Gariani K, Tran C, Phillippe J. Diabetes and cancer: an injurious association. Rev Med Suisse. 2010;6:1193-1194, 1196-1198.

47. Bonuccelli G, Tsirigos A, Whitaker-Menezes D, et al. Ketone and lactate "fuel" tumor growth and metastasis: evidence that epithelial cancer cells use oxidative mitochondrial metabolism. Cell Cycle. 2010; 9:3506-3514.

48. Hughes P, Bouillet P, Strasser A. Role of Bim and other Bcl-2 family members in autoimmune and degenerative diseases. Curr Dir Autoimmun. 2006;9:74-94.

49. Raun Q, Zhen SJ, Palmer S, et al. Roles of Bcl-3 in the pathogenesis of murine type 1 diabetes. Diabetes. 2010;59:2549-2557.

50. Rosenzweig M, Rosenthal CA, Torres VM, et al. Development of a quantitative assay to measure EBV viral load in patients with autoimmune type 1 diabetes and healthy subjects. J Virol Methods. 2010;164:111-115.

51. Rea D, Fourcade C, Leblond V, et al. Epstein-Barr virus latent and replicative gene expression in post-transplant lymphoproliferative disorders and AIDS-related non-Hodgkin's lymphomas. Ann Oncol. 1994;5(Suppl 1):S113-S116.
Clinical Ophthalmology

\section{Publish your work in this journal}

Clinical Ophthalmology is an international, peer-reviewed journal covering all subspecialties within ophthalmology. Key topics include: Optometry; Visual science; Pharmacology and drug therapy in eye diseases; Basic Sciences; Primary and Secondary eye care; Patient Safety and Quality of Care Improvements. This journal is indexed on

\section{Dovepress}

PubMed Central and CAS, and is the official journal of The Society of Clinical Ophthalmology (SCO). The manuscript management system is completely online and includes a very quick and fair peer-review system, which is all easy to use. Visit http://www.dovepress.com/ testimonials.php to read real quotes from published authors. 